\title{
Téoros
}

Revue de recherche en tourisme

\section{Le Québec est un grand parc : un domaine à exploiter ou à conserver?}

\section{Jean Pelletier}

Volume 3, numéro 2, juillet 1984

L'intervention étatique dans le domaine du tourisme : premiers

coups de sonde

URI : https://id.erudit.org/iderudit/1080803ar

DOI : https://doi.org/10.7202/1080803ar

Aller au sommaire du numéro

Éditeur(s)

Université du Québec à Montréal

ISSN

0712-8657 (imprimé)

1923-2705 (numérique)

Découvrir la revue

Citer cet article

Pelletier, J. (1984). Le Québec est un grand parc : un domaine à exploiter ou à

conserver ? Téoros, 3(2), 24-29. https://doi.org/10.7202/1080803ar d'utilisation que vous pouvez consulter en ligne.

https://apropos.erudit.org/fr/usagers/politique-dutilisation/ 


\title{
Un domaine à exploiter ou à conserver?
}

\author{
par Jean Pelletier
}

Une superficie totale de $1,024,184 \mathrm{~km}^{2}$ pour certains, de $1,540,681 \mathrm{~km}^{2}$ pour d'autres! Nous ne sommes pas à 100,000 milles carrés près au pays du Québec. Nous avons de l'espace. Comme on nous l'enseigne, cela représente une superficie trois fois supérieure à celle de la France et 7,2 fois celle de la Grande-Bretagne. Ou si vous préférez la comparaison américaine, cela équivaut à la superficie réunie des Etats de New York, du Vermont, du New Hampshire, du Maine, du Massachusetts, du Rhode Island, du New Jersey, de la Pennsylvanie, de la Californie... et du
Texas. Et contrairement à la plupart des pays occidentaux, l'Ėtat québécois est encore propriétaire de $90 \%$ de son territoire. ${ }^{111}$

On parle du Québec comme du pays du million de lacs. II faudra apprendre à compter : selon des amateurs d'eauxvives, il y a uau Québec 100,000 lacs importants, et, si l'on compte le moindre étang, 1,600,000. Quant aux rivières, si Fon compte jusqu'au plus petit ruisseau, on en estime le nombre à 6,000,000 loui, vous avez bien $/ u$, six millions/w ${ }^{|2|}$. Que de façades maritimes! Uniquement dans
I'archipel de Montréal, il y a plus de 300 îles et plus de $1,000 \mathrm{~km}$ de rives.

De Ville-Marie à Lourdes-de BlancSablon, de Coaticook à Ivujivik, c'est beau, c'est grand. Mais impossible de tout voir. Des érablières du sud à la taïga du nord, il y en a pour tous les gouts : il suffit d'en avoir les moyens.

Le seul problème, c'est que nous sommes moins de sept millions à nous partager cet espace, à exploiter ses ressources, à penser à en profiter maintenant tout en

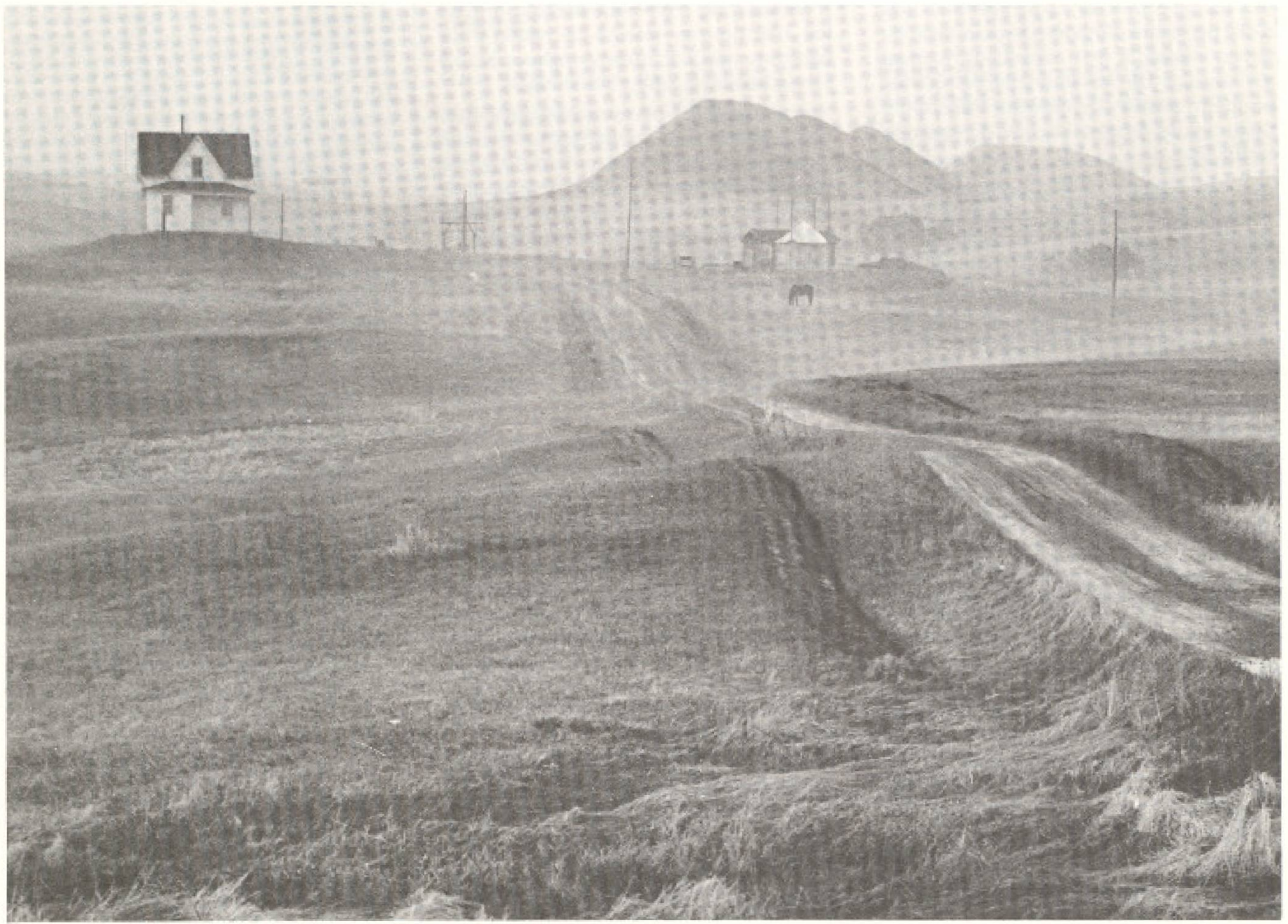

Un territoire immense. . . et souvent sublime de l’Outaouais aux lles de la Madeleine. Sur la photo: Pointe-Basse aux lles de la Madeleine. 
étant convaincus qu'il en restera presqu'autant pour ceux qui vont nous survivre. A preuve les petites communautés autochtones éparpillées aux quatre coins depuis des lunes.

Qui plus est, la très grande majorité d'entre nous avons choisi de vivre le long du Saint-Laurent, un des grands fleuves du monde dont le débit (à Montréal) est de 8,500 mètres cubes à la seconde pendant qu'à la hauteur de l'íle d'Anticosti, ses rivages sont éloignés de $125 \mathrm{~km}$.

Avec notre immense territoire, nos saisons climatiques bien découpées, nos espaces quasi illimités de chasse et de pëche, nous faisons des envieux du côté des vieux pays comme chez nos voisins americains qui arrivent tous mal à croire qu'en 1983, le troupeau de caribous de notre toundra qui se déplaçait en petits groupes, formait une colonne de $40 \mathrm{~km}$ de large sur $90 \mathrm{~km}$ de long $(500,000$ têtes $)$.

Rien d'étonnant à ce qu'on s'étonne de nous voir s'acharner a faire des plans d'urbanisme et des schemas d'aménagement, à créer des réserves fauniques ou écologiques, à déclarer des territoires arrondissement naturel, à imaginer des plans directeurs pour la conservation et la mise en valeur de sites.

Plus d'un (Français, par exemple) s'émerveille de nous voir, jour et nuit, remodeler la nature, détoumer de puissants cours d'eau, aménager des routes qui éventuellement, formeront dans la zone subartique, un passionnant domaine touristique à une heure de jet de Montréal ${ }^{3 !}$.

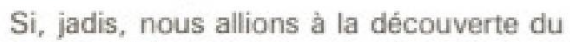
pays habité par les chemins de campagne bordant les terres cultivées, nous avons maintenant des autoroutes qui vont dans toutes les directions vers les lacs lotis pour les villégiateurs, les centres de ski ou les parcs pour les amateurs de camping.

II suffit de survoler la petite portion de notre territoire un tant soit peu peuplé (de Hull à Sept-îles et de Valleyfield à Rimouski) pour reconnaître nos aires de jeu : golfs, marinas, terrains de balle et de tennis, piscines familiales, etc. II n'y a pas une seule ville au monde autre que Montréal qui peut offrir à ses résidents autant de plans d'eau pour pratiquer les activités de nautisme. Notons que c'est quand même un milieu un peu trop pollué pour la baignade....

Qu'avons-nous a faire avec les notions d'aménagement du territoire et de cadre organisé de l'espace?

Qu'avons-nous à faire d'une approche de développement du tourisme quil doivent tenir compte de contraintes?

Les Américains sont toujours venus chasser sur nos terres, passer une partie de l'été à Murray Bay (La Malbaie pour les mal initiés). Quant aux familles québécoises, plusieurs ont leur résidence privée de villégiature, les moins nanties se conten- tant d'une roulotte ou d'un camp de pêche! ${ }^{(4)}$

Voilà le terrain de jeu des techniciens et technocrates chargés d'améliorer notre cadre de vie. Et, pendant qu'ils assument leur portion de pouvoir, l'État nous suggère d'aller jouer dehors!

\section{L'État dans tous ses états}

Le Quebec compte à peine dix habitants au mille carré. On peut imaginer l'ampleur des difficultés que rencontre le planificateur (politique ou technocratique) dont la tâche consiste á aménager un territoire comportant une aussi faible densité.

Le fonctionnaire du ministẻre des Affaires culturelles (MAC) fait des inventaires de paysages architecturaux, celui de l'Agriculture, des Pêcheries et de l'Alimentation (MAPAQ) une étude-cadre sur la construction d'aboiteaux, celui de l'Énergie et des Ressources (MER) un manuel de confection du plan de gestion des terres publiques, celui de I'Environnement (MENV/Q) un atlas des espaces verts, des sites naturels et des réserves écologiques; celui du Loisir, de la Chasse et de la Peche (MLCP) des normes génerales pour l'aménagement intégré de la faune et de la forêt et celui de l'Industrie, du Commerce et du Tourisme (MICT) une programmation des aménagements $d^{\prime}$ une station comme Magog-Orford.

Quel ministère n'a pas sa loi let ses règlements d'application) pour gérer, avec ses lunettes, sa partie du territoire? Le MAC avec la Loi sur les biens culturels (1972), le MENVIO avec la Loi sur les réserves écologiques (1974), le MLCP avec la Loi sur les parcs (1977) et la Loi sur la conservation de la faune (1969 et revue en 1983 pour $y$ ajouter at de la mise en valeurs) Et le pauvre ministre qui doit écouter (et respecter?) les avis d'une Commission des biens culturels, d'un Conseil consultatif de l'envirannement, d'un Conseil de la faune.

Par prudence, nous n'avons pas parlé jusqu'ici de la Loî sur la qualité de l'environnement (1972, revue en 1978) et du Bureau d'audiences publiques sur l'Environnement, de la Loi sur la protection du territoire agricole (1978) et de la Commission (CPTA) qui en découle, de la loi 125, Loi sur l'aménagement et sur l'urbanisme (1979) et de la Commission nationale de Iaménagement qui, selon l'article 221 de la loi \&donne des avis sur la conformité d'un plan d'urbanisme ou d'un règlement de zonage, de lotissement ou de construction aux objectifs d'un schéma d'aménagement et aux dispositions du document complementaire d'une municipalité regionale de comté, sur la conformité d'un reglement de zonage, de lotissement ou de construction au plan d"urbanisme d'une municipahté, ou sur la conformité d'une intervention gouvernementale aux objectifs dum schéma d'aménagement et aux dispositions d'un reglement de contrôle interimaires.

Heureusement qu'il n'y a que $94 \mathrm{MRC}$ en plus des Communautés urbaines de Montréal (CUM) et de Québec (CUQ) et régionale de l'Outaouais (CRO).

Soulignons quand même en passant que, de son côté, le ministre de l'Environnement peut, en vertu de l'article 2 de la Loi sur lá qualité de l'environnement, «al coordonner les recherches qui sont faites par les ministères et organismes du gouvernement sur les problemes de la qualité de l'environnement; b) exécuter ou faire exécuter des recherches, études, enquêtes et inventaires sur tout ce qui concerne la qualité de lenvironnement; c) elaborer des plans et programmes de conservation, de protection et de gestion de Venvironnement... ta tandis que, du sien, la CPTA a pour mandat de s'assurer que tous les sols propices a l'agriculture soient réservés à cette fin ${ }^{15 !}$.

Alors si le ministre d'État au Développement économique se met à nous parler de l'uaménagement spatial du produit touristiquen ${ }^{[6]}$ pendant que son collègue délégué à l'Aménagement et au Développement régional nous propose d'aménager l'avenir en faisant Le choix de régions ${ }^{(7)}$, il est presque normal de perdre son francais quand en 1984 , entre l'énoncé de deux normes de la Commission de toponymie, chargée de l'aménagement linguistique, on voit le MAPAO souligner par un dépliant que l'agriculture, la faune et l'environnement ase voisinent depuis toujours; et le MENVIQ produire une affiche valorisant la protection de l'ail des bois...

Surtout, ne perdons pas de vue que c'est le MER qui est responsable de la gestion des terres publiques. "Les terres publiques, en tant que support des multiples ressources naturelles du milieu et des activités que s'y deroulent, revetent une importance capitale et apparaissent comme un instrument privilégié d'aménagement rational et de développement du territoires ${ }^{(8)}$. Le MER met d'ailleurs l'épaule à la roue avec sa politique d'accessibilité par la location de terrains de villégiature résidentielle et ses centres éducatifs forestiers à ne pas confondre avec les réserves écologiques (MENVIQ), fauniques et les parcs de conservation (MLCP). II la met également bien sûr dans la rationalisation et le développement du secteur de la transformation des bois les forêts publiques couvrent prés de $90 \%$ du territoire forestier et foumissent $80 \%$ de la récolte annuelle et dans le développement de I'exploitation des ressources minérales.

Par une plus grande prudence, nous n'avons pas parlé non plus jusqu'ici de l'autre niveau de gouvernement, quil, visà-vis de ses interlocuteurs de provinice, 
entretient quelques velléités d'intervention directe.

Pensons, par exemple, à Parcs Canada (Environnement Canada) qui aménage et administre deux grands et magnifiques parcs dits nationaux (Forillon et de la Mauriciel, restaure une treizaine de parcs et lieux historiques (du fort Chambly aux Forges du Saint-Maurice) et quatre canaux historiques.

Pensons aussi au Service canadien de la faune (Environnement Canadal qui a encouragé (depuis 1916) l'établissement au Québec de 22 refuges d'oiseaux migrateurs et, depuis 1977, aménagé 6 réserves dites nationales de faune. Signalons d'ailleurs une récente petite coilncidence : au cours de l'automne 1983, le MLCP a vu sa Loi sur la conservation de la faune amendée entre autres pour prévoir la creation d'un fonds spécial pour l'achat et l'aménagement d'habitats menacés; or en fevvier 1984, Ottawa annoncait Idiscretement) la création d'une nouvelle société de la Couronne, Habitat faunique Canada, intéressée par l'acquisition de tout habitat menacé de disparition : selon un porteparole fédéral, ale problème le plus urgent demeure la degradation rapide des terres humides, endroits de prédlection pour la sauvagine; ce sont les marais de la vallée du Saint-Laurent au Quebec.... qui retiennent particuherement /attentions ${ }^{191 .}$

C'est le groupe privé Canards Illimités Canada qui va se réjouir de voir nos deux niveaux de gouvernement unir leurs efforts (chacun de leur côtél) pour épauler le travail entrepris par l'organisme en 1937.

Un autre bel exemple de complémentarité fédérale-québécoise est l'Outaouais. D'un côté, signalons les interventions de la Commission de la Capitale nationale
(CCN) créée en 1958 qui, en vertu de la loi qui la régit, peut entre autres réaliser avec les municipalités des projets conjoints, construire des parcs, sauvegarder des sites historiques, organiser des activités culturelles et a accomplir tout ce qui se rattache a ses objectifs ou contribuer à accomplir son mandats ${ }^{(10)}$ et dont le territoire couvre, au Québec, plusieurs municipalités et le parc de la Gatineau. De l'autre, la Société d'aménagement de I"Outaouais (SAO) a pour mission de favoriser l'essor économique de la région et de réaliser des projets d'équipement industriel, commercial, récréatif et touristique ${ }^{1111}$.

Voilà quelques-uns des exemples qui permettent de croire que, finalement, tout n'est pas si simple du point de vue de la gestion du territoire. Le Québec, un grand parc? Ou une usine à pâtes et papier, à

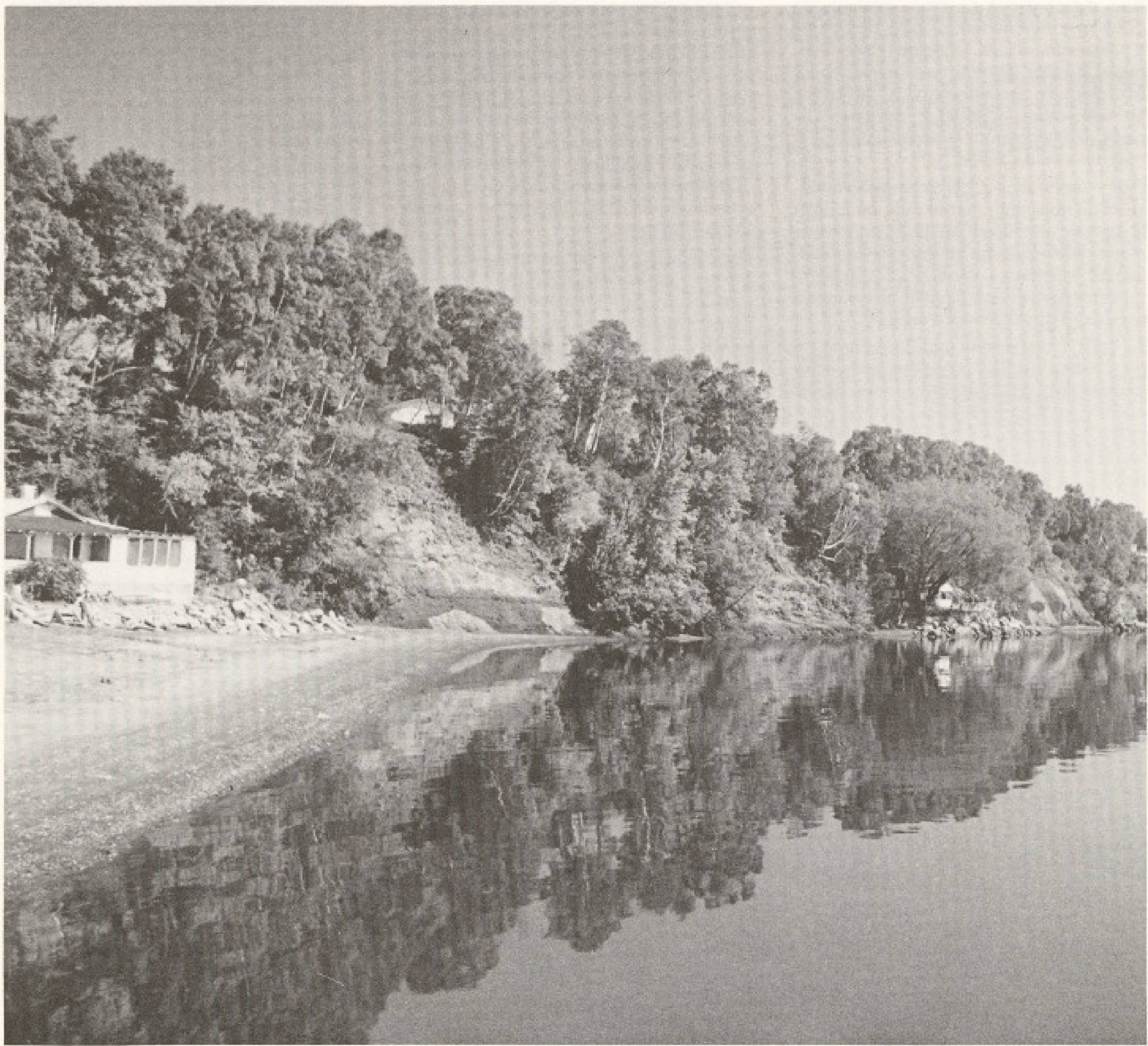

L'exemple de la villégiature est un intéressant symbole de notre rapport avec l'environnement. 
b́lectricité? Un grand champ destiné à la production agricole? Une réserve de chasse et de pêche?

Pratiquement tous les gens d'ici sont des riverains du système du Saint-Laurent : les trós millions de résidents de la région de Montréal qui connaissent les Laurentides imaginent connaître l'espace nordique. Géographie pour géographie, les plages du Maine et de la Floride. comme les berges de la Seine, sont plus faciles à vendre aux voyageurs québécois que nos chemins d'eau et nos lointains arrière-pays.

\section{Tourisme et espace : une dialectique subtile}

De toutes les activités socioéconomiques, le tourisme est probablement l'une de celles qui devraient entretenir une relation tres intime, voire organique, avec I'environnement. Contrairement aux exploiteurs de ressources naturelles, industriels comme producteurs agricoles, les promoteurs touristiques, I'État compris, dépendent (théoriquement!) de la qualité du milieu, de sa préservation et de son aménagement. Si non de quoi sera composé le paysage de l'offre? D'un casino ou d'un lieu de pèlerinage?

L'immensité de notre territoire, la conviction du caractère inépuisable de nos ressources ont historiquement encouragé les promoteurs, privés et publics, à administrer le domaine québécois comme si chaque citoven pourrait toujours trouver son compte dans le calme naturel de asons lac et de asons plan d'eau. Les deux millions d'insulaires qui vivent sur lîle de Montréal savent maintenant que leurs rives sont pratiquement inaccessibles. Dans les rares cas où les berges ne sont pas privatisées, ils ont accès a une eau généralement impropre à la baignade. Plus d'un en est rendu à souhaiter le prolongement de l'autoroute des Laurentides jusqu'à Mont-Laurier pour ouvrir de nouveaux centres de villégiature pour le bénéfice des urbains en mal de verdure et d'eau de qualité.

A côté des amateurs, plus ou moins privilégiés, de chasse et de pêche Ivoir encadré, p. 28), l'exemple de la villégiature est un intéressant symbole de notre rapport avec l'environnement. II n'y a pas un demi-siècle que nous avons quitté massivement nos terres pour venir tenter de survivre en ville. Et nous avons tout aussi massivement occupé toutes les berges des lacs et rivières dans un rayon de $200 \mathrm{~km}$ de Montréal, de Hull, de Québec et de Trois-Rivières pour s'oxygéner le temps du week-end. Les défenseurs des propriétaires villégiateurs font même la preuve de leur importance économique et de leur souci environnemental en soulignant que wle commerce des installations septiques frise les 40 milhons de dollars annuellementy $y^{1121}$.
Des analystes ont entrepris récemment de se pencher sur le phénomène des résidences secondaires : il n'est pas que québécois évidemment, mais combien développé ici. aLa justification proposee pour expliquer cette nouvelle forme d'exode est Ialinéation dont témoignent nos villes et notre rythme de vie... elle répond cependant d'une mode et meme d'un certain snobisme en manifestant la réalisation d'aspirations plutot que de besoins ${ }^{133}$.

II n'est pas étonnant dans ce contexte que la psychiatrie gouvernementale s'interroge: rle développement anarchique et des. tructeur des agglometrations urbaines, la proliferation des résidences secondaires dans les regions de loisir et de detente reflètent une confusion des valeurs... Comment, au cours de notre courte histoine. avons-nous pu passer d'une situation de maitrise de notre espace à une autre de colonisation et d'occupation de ce mëme espace? $y^{14}$

Après plusieurs années de développement dans toutes les directions et sous toutes ses formes de nos ressources touristiques [paysages, faune et autres attraits], $\pi^{\prime} y$ $a-t-i l$ pas lieu de s'interroger sur les finalités des réalisations que nous avons encouragées ou tolérées jusqu'ici? Quoi de plus enormals que de vouloir accueillir des touristes-clients; mais quoi de plus souhaitable que de leur donner le goût de revenir en leur en offrant les conditions de base. L'État a-ț-il une responsabilité dans la sauvegarde et l'amenagement de nos espaces au-delá de la création d'une réserve écologique à la Pointe-Heath (extrémité est d'Anticosti) ou du parc d'Aiguebelle (Abitibi)?

Notre environnement immédiat est largement construit. L'Etat commence cependant à manifester des signes évidents de préoccupation : restauration de vieux ports à Montréal comme à Québec, réaménagement du VieuxMontréal. Nous sommes tant bien que mal partis à la reconquête de nos milieux. édifies. Des sommes importantes sont maintenant consacrées à un projet d'aménagement des eaux de la région de Montréal, Peut-butre verrons-nousemerger le projet du début des années 70 kun fleuve, un parcs...

Les grandes législations récentes sur l'aménagement et l' urbanisme témoignent d'un souci d'organiser le présent pour préserver l'avenir. Écoutons le père de la loi 125: wle Québec possède des caractéristiques spéciales. Fondamentalement, c'est un territoire neuf. On est arrivé dans des espaces si vastes, quion n'a jamais senti le besoin d'économiser le territoire, on en développait des parcelles par nécessité. Maintenant, c'est different... Il y a tout de suite une notion d'economie d'espace qui intervient et nécessairement des perspectives d'aveniry ${ }^{15}$.
Malgré la multiplication des actes législatifs et réglementaires, malgré les très nombreuses mesures gouvernementales affectant la conservation et l'utilisation du territoire, il est loin d'être évident que nous avons une vision claire que ale developpement touristique doit, dorénavant, etre considere comme l'un des eléments d'un système phs vaste comprenant utourismeloisir de plein air et conservation de la nature ${ }^{16}$.

Depuis 50 ans, nous avons trop souvent laissé chacun occupé un espace, usons terrain, sans toujours mesurer les effets d'agression sur le milieu, milieu support et milieu d'accueil. II ne faut plus seulement évaluer la situation du tourisme par le calcul alambiqué des retombées des depenses directes et indirectes des woyageurs. II faut davantage valoriser l'importante nécessité pour l'État de veiller au grain en confirmant la volonté d'une planification spatiale qui privilegie le souci de façonner nos espaces pour plus d'un usage, pour plus d'une clientèle et pour plus d'une génération.

II ne suffit donc plus qu'à identïtier les objectifs, les espaces, les conditions et les moyens! L'État doit intervenir mais sur quoi, quand et comment?

\section{Un cas d'espèce : le ministère du Loisir}

Le ministêre du Loisir, de la Chasse et de la Pêche (MLCP) est, comme son nom ne l'indique pas, notre véritable ministère du tourisme.

Savez-vous qu'en plus d'administrer des parcs (de récréation et de conservation) et des réserves fauniques, le MLCP possède et gère des camping, des plages, un jardin zoologique, des auberges, des golfs, un aquarium, des parcs nautiques, un village historique (Val-Jalbert), des chalets, le presbytere de Batiscan et combien d'autres équipements récréo-touristiques. Uniquement dans les secteurs de la faune et de la récréation dite de plein air, le MLCP a une allocation budgétaire annuelle de plus de 80 millions de dollars pour exploiter un vaste réseau de parcs, de réserves et d'établissements touristiques et récréatifs.

Mais ce $n^{\prime}$ est pas tout! Qui administre le Mont Sainte-Anne et loue à des concessionnaires le fond de terrain du MontTremblant et du Mont-Orford? C'est le ministère responsable de la politique de ski alpin, le MLCP. Ce n'est pas parce qu'une nouvelle situation concurrentielle dans le nord-est américain s'est développer que le Quebec n'est plus dans la coursel17h. Des investissements majeurs ont été faits en 1983 au Mont Sainte-Anne pour installer des canons à neige, une entente a été signée avec un nouveau promoteur pour assurer la relance du Mont-Tremblant et le ministre du Loisir vient d'annoncer une subvention de $3.5 \$$ millions à Gestion 
Orford ${ }^{(19)}$. Et signalons, entre autres, les coùteuses etudes en cours concernant le site de Petite Riviere Saint-François (Charlevoix).

C'est du même ministêre que relève, dans le cadre des lois sur la faune ou sur les parcs, la gestion de nombreux el importants territoires conjointement avec les gestionnaires des zecs et les quelques 700 pourvoyeurs. A ce titre, le ministère est obligé d'avoir ă son service quelques douzaines d'experts pour édicter des normes (qui peut chasser ou pêcher, lieux, périodes, quantitél et quelques centaines d'agents de la paix pour les faire respecter.

Ce $n^{r}$ est donc pas pour rien que le MLCP mène depuis des années des campagnes publicitaires nous recommandant d'aller jouer dehors. Ce n'est peut-être pas pour rien qu'un projet de loi vient d'étre déposé à l'Assemblée Nationale pour confier à une future aSociété des établissements de plein air du Québeck la gestion d'équipement actuellement gérés par le MLCP. Le projet de loi ne devant ëtre adopté qu'á l'automne, les intéressếs auront quelques semaines pour connaitre et comprendre la nature et la portée de cette action. Si l'énoncé ministériel préliminaire souligne les avantages d'une gestion plus souple et d'une diminution espérée des coûts d'exploitation, il faut aussi s interroger sur les fondements d'une telle initiative. Le Gouvernement aurait pu décider de céder, de vendre, de louer des concessions ou d'imaginer des formules mixtes en association avec des intervenants publics ou privés. S'agit-il d'une simple technique de dégraissage de l'appareil public ou d'une nouvelle approche de gestion publique? Le futur holding aPlein Air Québecs va-t-il rêussir ă concilier responsabilité collective et rentabilite financiere?

Comme nous l'avons vu plus haut, le MLCP n'est pas le seul ministère sectoriel à assumer sa part de responsabilité des ressources patrimoniales, Ce rest pas parce que le MLCP voulait faire un parc de I'archipel de Mingan, déjà classé arrondissement naturel par le MAC, que le gouvernement fédéral s'est privé de faire une offre d'achat plus substantielle au détenteur des droits de propriété.

Ce n'est pas parce que le MLCP propose un intéressant plan directeur pour la conservation et la mise en valeur du lac Saint-Pierre (1983) que les agriculteurs et le MAPAO ne souhaitent plus réaliser l'endiguement de leurs terres" ${ }^{(19)}$. On avait déjà vu des situations d'intérêts confictuels a l'occasion de la bataille des aboiteaux de Kamouraska. On en verra d'autres où le difficile équilibre entre la protection de l'écosystème et les intérêts des producteurs fera l'objet d'une bataille, à défaut d'une concertation.

Ce qui est heureux mais exigeant, c'est que de nombreux mécanismes ont eté crées pour permettre aux groupes et aux

\section{Chasse, pêche, zec et pourvoirie}

C'est en 1883 que commence officiellement la location des cours d'eau à des clubs sportifs incorporés. Les revenus de location totalisent 56,226 s en 1901 , dont 46,537 \& de draits de pëche ${ }^{17}$. En contrepartie, 16,030 \$ étaient dépensés pour la protection du gibier cette année-là. Voilà déjă une politique efficace de protection du cheptel tout en générant des revenus à l'État!

Dès cette époque, plusieurs clubs de pêche au saumon sont créés. En 1899, on dénombrait aussi plus de 30 clubs dans les Laurentides et 70 quinze ans plus tard. $80 \%$ des membres de ces clubs sont des anglophones souvent associés aux compagnies forestieres et minières.

A partir de 1905, la consolidation du système des clubs évolue : le cercle des locataires des territoires gagnent les résidents du Quebec dont le nombre s'accroitt régulièrement jusqu'en 1945 pour ensuite monter en flèche jusqu'en 1960 186\% des effectifs).

Ces memes quinze annees virent passer le nombre de locations de 615 à 1,908 , pour la plupart au service des clients et des électeurs du gouvernement du temps. C'est ả la même époque que s'instaure le svstème des baux commerciaux (pourvoirie) et celui du . . braconnage puisque les meilleurs territoires sont loués.

A compter de 1946, la Fédération des associations de chasse et pêche du Québec (FACPOI est creee : elle devra rapidement composer avec l'accroissement de la pression populaire. La fédération recommande donc au gouvernement la création d'endroits publics de pêche dans chaque région, ucertains lacs ou rivières qui ne sont pas sous bail et à proximité des villes: ${ }^{|2|}$.

\section{Le adéclubage»}

Au début des années 60 , des actions revendicatrices sont menées en Mauricie, sur la Cöte-Nord. En 1961, 32 contestataires sont arrètés sur la rivière Matapédia : ils réclamaient la révocation d'un bail consenti à un club américain.

Une vaste opposition au système des droits privés de location va se développer: création du Mouvement pour l'abolition des clubs privés sur les territoires de la Couronne, Mouvement pour la récupération des rivières à saumon du Québec, avec le soutien du milieu syndical de la CSN.

L'engagement public du chroniqueur Serge Deyglun comme les déclarations de quelques haut-fonctionnaires vont dans le sens d'une transformation du système. Mais les résistances sont nombreuses et fortes malgré les discours politiques favorables à la démocratisation. Les luttes s'intensifient et se radicalisent à la fin des années 60 : les contestataires occupent les territoires.

Ce n'est cependant qu'en 1977 que le gouvernement abolit les droits exclusifs des clubs privés de chasse et de péche $e^{|3|}$ et crée des zecs gérées par des associations d'utilisateurs. Aujourd'hui il $\gamma$ a 67 zecs-faune et 6 zecs-saumons (12 rivières accessibles à tous). L'action gouvernementale de 1977 a créé un mouvement: irrẻversible favorable à la démocratisation et ce, sans entrainer des coûts énormes.

Plus d'un observateur s'inquiètent cependant de l'évolution actuelle : les asquatters» continuent à occuper des territoires, les responsables de zecs ont des moyens limites pour gérer la ressource faunique, d'anciens clubs continuent de façon déguisée, des pourvoiries opèrent illégalement, la majorité des rivières ả saumon ne sont pas encore «déclubéesw ${ }^{|4|}$.

Comment assurer à la fois une gestion collective de la ressource et une rentabilité des opérations sans tolérer l'appropriation des territoires par des groupes minoritaires au détriment de l'accessibilité pour tous? Aux dernières nouvelles, il y a 600,000 chasseurs et 1,3 million de pêcheurs au Québec ${ }^{\text {ISt }}$. Comme l'a déjâ écrit un pêcheur inconnu : aj'ignore qui a découvert l'eau, mais ce n'était certainement pas un poissons.

(1) Les donnfes historiques et statistiques sont toutes tiress d'un momoire de maitrise Iscience politiquel : DESJAADINS, Jacques La politique de concession des droits prives de location en matiére de chasse et de péche au Québec do 1960 a 1977, Université de Montréal, février 1983, $170 \mathrm{p}$.

(2) La FACPO devient la Fédération québécoise de la Faune (FaF) en 1965.
(3) Les propriétaires de chalets et de camps ont conserwe la propriété de leurs actifs.

(4) Voir a ces sujets la récente plate-forme de revendications de la CSN, Nos loisirs et nos vacances. c'est pas du luxe!, awril 1984,75 p.

(5) Sentier chasse-peche, juin 1984, p. 10. 
citoyens de faire valoir leurs points de vue. Il en va ainsi en matière de parcs (création, modification ou abolition) où le ministre est tenu légalement d'entendre les intéressés (article 2 de la loi).

Espérons cependant que l'apprentissage de l'exercice de ces mécanismes de participation ne sera compliqué par la tendance, hélas souvent répétée, de l'appareil tant politique que bureaucratique de respecter le moins possible ses propres obligations. Sinon, pourquoi et pour qui planifier et aménager?

Ces aspects, en bonne partie positifs, ne doivent pas nous faire perdre de vue que le MLCP s'est historiquement préoccupé de la conservation des territoires éloignés des centres densément peuplés. Ce n'est pas avec les parcs du Mont Saint-Bruno et Paul-Sauvé (Oka), d'ailleurs ayant officiellement le statut de réserves de chasse et de pêche (sic), que la population métropolitaine peut s'uépivarders : les carences en milieu urbain sont importants; c'est la plutôt qu'à l'île Bonaventure que l'État québécois devrait travailler à produire un environnement mieux équilibré.

\section{Un pays à bâtir}

On a wfêté» en 1983 le centenaire de l'intervention de l'État québécois en matière de pềche sportive. Comme l'État fédéral n'est pas en reste, les gestes publics d'aménagement ont finalement une longue histoire. Mais le Québec des vingt derniêres années a formé progressivement de nouveaux experts qui, à leur tour, ont inventé un nouveau langage. Depuis I'adoption de la loi 125 , on a l'impression que quelques milliers d'élus locaux refont leurs classes. Les questions d'environnement sont devenues des préoccupations quasi permanentes. Qui plus est. l'aménagement est percu ou voulu comme un processus d'ordre politique : il va y avoir des tiraillements entre technocrates et élus, entre investisseurs et groupes de citoyens pour mesurer la compatibilité des objectifs de développement des uns et des autres.

$\mathrm{Et}$, comme au Québec on aime les handicaps, on ajoute aux exigences des approches sectorielles la difficulté résultant de l'absence d'un cadre de référence spatiale homogène: remarquons que plus d'un s'en tire fort bien par ailleurs, mais au détriment de qui?

L'Ėtat québécois, pour un, touche à tout, tantôt avec une volonté marquée d'intégrer dans ses projets et ses approches de développement un souci de planification à caractère écologique, tantôt avec le sentiment urgent de garantir au premier promoteur wenu une liberté d'intervention sans mesures restritives.
L'État défenseur et promoteur du bien collectif public est pris entre quatre ou cinq feux gouvernementaux: le contrôle de l'excédent des dépenses, les emplois du lendemain, les exigences multiples des groupes d'intếrêt, les verdicts électoraux du surlendemain, le patrimoine des prochaines générations.

Les Québécois voudraient avoir le plus beau territoire du monde : les palmiers de Charlebois et la Basse Cote-Nord de Vigneault. L'État québécois veut satisfaire ses citoyens, écologistes comme développeurs, et séduire les étrangers, touristes comme investisseurs. L'État est nécessairement juge et partie quand il légifère, acquiert ou aménage pour préserver et offrir des services. Si l"Ëtat doit continuer à s'occuper de la gestion du territoire, il vaudrait mieux savoir à quelle(s) fin(s).

Récemment un auteur soulignait que la prudence et la concertation étaient les deux vertus de l'aménagement touristique québécois grâce aux interventions législatives et réglementaires récentes ${ }^{(20)}$. Espérons qu'il ne s'agit pas d'une croisée de chemins entre le blocage et la dynamisation de la spécificité d'un pays, peuple et territoire mieux intégrés.

L'État-propriétaire, comme l'Étatentrepreneur, a un monde à recréer. L'État-providence est en crise. Le pays providentiel est en friche. $f$

\section{Notes et références}

(1) Ministere de l'Energie et des Ressources, Rapport annuel 1980-1983, Québec, p. 49.

(2) Fédération québécoise de canot-kayac, Guide des rivières du Québec, Editions du Jour, 1973, p. 262.

( 3) MARTIN-TARD, L., Au Québec, Guides bleus, 1976, p. 207.

(4) Une famille québécoise sur cinq possède un chalet ou une roulotte; GIRARD, M., La Presse, 15 mai 1984.

(5) "Tout usage autre qu"agricole est interdit sur une terre située dans la zone agricole. II n'est doné pass pirmis d'y établir un commerce, de construire une industrie ou tout autre batiment qui ne sert pas directement a l'agriculture et a l'agriculteuro nous précise une brochure de renseignements gúnéraux du MAPAO, 1979 p. 22. Sauf exception, bien sūr ....

(6) Bátir le Québoc, Énoncé de politique économique, Gouwamement du Québec, 1979, p. 134 ,
(7) Document de consultation sur le développement des rógions, Gouvernement du Québec, 1983, $132 \mathrm{p}$.

(8) Ministöre de l'Energie et des Ressources. Rapport annuel 1982-1983, Québec, p. 49.

(9) MOUNTAIN, B. sous-ministre, Environnement Canada, La Presse, 3 mars 1984.

(10) Commission de la Capitale nationale, Rapport annuel $1990-1981$, p. 45.

111) La SAO est una corporation créée en vertu du titre III de la Loi de la Communauté régionale de I'Outaouais. De par cente lol, la Société jouit des droits at privilèges d'un mandataire du Gouvernement du Québec.

Le territoire confie a la Société comme champ d'action couwre 34,260 kilomètres carrés dans I'Outaouais.

(12) FAPEL, La villégiature une force teconomique, 1983, P. 2.

(13) ST-AMOUR, J.-P.. La villégiature au Québec, problématique de l'aménagement du territoire, Editions Asticou, 1979, p. 7 .
(14) La politique québécoise du développement culturel, Gouvernement du Québec, 1978, p. $159-160$.

(15) LEONARD, J, ministre d'État à l'Aménagement, Développement Québec, vol. 6. no 1 , mars-avril 1980 , p. 19

(16) CAZES, G. LANQUAR, $R_{1}$, et RAYNOUARD, $Y$. L'Aménagement touristique, Presses universitaires de France, 1980, p. 7.

(17) La politique québécoise pour le ski alpin, MLCP, 1983, 58 p.

(18) La presse, 13 juin 1984.

(19) Consultation publique : synthese des mémoires, MLCP et COLASP, novembre 1983, $78 \rho$.

(20) ARCHAMBAULT, $Y$. Des outils ef des intervenants. Téoros, vol. 2 no 3 , octobre 1983 . p. 6-7. 